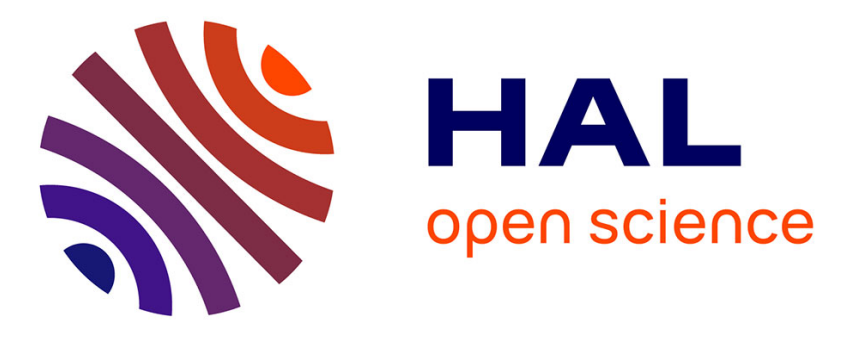

\title{
Selective Area Growth of GaN Nanowires on Graphene Nanodots
}

Martina Morassi, Nan Guan, Vladimir G Dubrovskii, Yuri Berdnikov, Camille Barbier, Lorenzo Mancini, Ludovic Largeau, Andrey Babichev, Vishnuvarthan Kumaresan, Francois H Julien, et al.

\section{To cite this version:}

Martina Morassi, Nan Guan, Vladimir G Dubrovskii, Yuri Berdnikov, Camille Barbier, et al.. Selective Area Growth of GaN Nanowires on Graphene Nanodots. Crystal Growth \& Design, 2019, 20 (2), pp.552-559. 10.1021/acs.cgd.9b00556 . hal-03048504

\section{HAL Id: hal-03048504 https://hal.science/hal-03048504}

Submitted on 9 Dec 2020

HAL is a multi-disciplinary open access archive for the deposit and dissemination of scientific research documents, whether they are published or not. The documents may come from teaching and research institutions in France or abroad, or from public or private research centers.
L'archive ouverte pluridisciplinaire HAL, est destinée au dépôt et à la diffusion de documents scientifiques de niveau recherche, publiés ou non, émanant des établissements d'enseignement et de recherche français ou étrangers, des laboratoires publics ou privés. 


\title{
Selective area growth of GaN nanowires on graphene nanodots
}

\author{
${ }^{\dagger}$ Martina Morassi, ${ }^{\dagger}$ Nan Guan, ${ }^{*}$ Vladimir G. Dubrovskii, ${ }^{*}$ Yuri Berdnikov, ${ }^{\dagger}$ Camille Barbier, \\ ${ }^{\dagger}$ Lorenzo Mancini, ${ }^{\dagger}$ Ludovic Largeau, ${ }^{*}$ Andrey Babichev, ${ }^{\dagger}$ Vishnu Kumaresan, ${ }^{\dagger}$ Francois $H$. \\ Julien, ${ }^{\dagger}$ Laurent Travers, ${ }^{\dagger}$ Noëlle Gogneau, ${ }^{\dagger}$ Jean-Christophe Harmand and ${ }^{\dagger}$ Maria \\ Tchernycheva \\ † Centre de Nanosciences et de Nanotechnologies (C2N), UMR9001 CNRS, University Paris \\ Sud, University Paris Saclay, 10 Boulevard Thomas Gobert, Palaiseau, France. \\ ‡ ITMO University, 197101 St. Petersburg, Russia.
}

Keywords: nanowires, graphene, SAG, nucleation, GaN, PA-MBE

\begin{abstract}
We present a new approach to achieve selective area growth of GaN nanowires by plasmaassisted molecular beam epitaxy. The nanowires are grown on graphene nanodots which are patterned by electron beam lithography from polycrystalline graphene patches transferred to $\mathrm{SiO}_{2}$ substrates. The GaN nanowires grow on these graphene nano-domains with a perfect selectivity with respect to the $\mathrm{SiO}_{2}$ surrounding surface. The results demonstrate that a single monolayer of graphene can withstand the lithography process without losing its ability to induce epitaxial growth. The nanowire length distribution and patterns' fill factor are analyzed in the framework of a theoretical model, which takes into account an incubation time dependent on the graphene dot size. Overall, these results represent the first demonstration of selective area nanowire growth on a regular array of graphene nano-domains.
\end{abstract}




\section{INTRODUCTION}

Nitrides nanowires (NWs) are promising nanomaterials for many optoelectronic applications, in particular for light emitting diodes (LEDs) ${ }^{1,2}$. Among several advantages, NW geometry helps to elastically accommodate large lattice mismatches. This offers the possibility to achieve highquality In-rich heterostructures ${ }^{3}$, which are difficult to fabricate in the form of thin films. Molecular beam epitaxy (MBE), with its relatively low growth temperature, has been able to produce green and red InGaN/GaN NW-based LEDs. ${ }^{4,5}$ However, in LEDs based on selfassembled NWs, emission wavelength and electrical injection suffer from strong inhomogeneities. ${ }^{6,7}$ To solve this problem, a selective area growth (SAG) approach of regular NW arrays must be implemented. Indeed, SAG yields a good control of the NW location with more uniform dimensions and composition within InGaN/GaN heterostructures. ${ }^{4,8-12}$

SAG of GaN NWs is generally performed on monocrystalline substrates promoting vertical growth of NWs. A nanopatterned mask layer is used to inhibit the growth outside the nanoopenings and thus to provide growth selectivity. Along these lines, there have been several reports on SAG of GaN NWs by plasma-assisted (PA) MBE. The first demonstration, by the group of Kishino, was performed at high growth temperatures on $\mathrm{Si}$ (111) substrates covered with Ti masks ${ }^{12-14}$. SAG of GaN NWs on Si (111) was further developed by other groups ${ }^{15-18}$ and on other substrates including diamond ${ }^{19}$ and sapphire ${ }^{20-22}$, with either $\operatorname{TiN}_{\mathrm{x}}{ }^{10,21,22}, \operatorname{SiN}_{\mathrm{x}}{ }^{17,18}$ or $\mathrm{SiO}_{\mathrm{x}}{ }^{15,16}$ masks. In most cases, the growth of an intermediate or template layer, such as $\mathrm{AlN}^{13,18,23,24}$ or $\mathrm{GaN}^{12,18,20,21}$ was necessary to promote NW nucleation in the mask openings and to reduce the density of crystallographic defects related to the lattice mismatch with the substrate. This represents an additional growth step to the preparation of the patterned substrates. Regarding the mask, a rough surface morphology may compromise the growth selectivity since imperfections may induce undesirable parasitic nucleation. An alternative SAG approach was proposed by Ishizawa et al. ${ }^{14}$ to overcome this issue. Instead of defining a mask to inhibit growth, the authors used nitridated $\mathrm{Al}$ nanodroplets on $\mathrm{Si}$ (111) to promote selective nucleation on AlN with respect to the Si surface. This approach was implemented with 20-nm-high AlN pedestals and tube-like structures were obtained due to a preferential nucleation of GaN NWs at the edges

of the pedestals. Here, we propose to use graphene, an ultimately thin crystalline material, to promote selective nucleation of GaN NWs. 
Recently, a specific case of van der Waals epitaxy combining NW crystals (e.g. InGaAs, GaN, $\mathrm{ZnO}$ ) having $\mathrm{sp}^{3}$ bonds with layered materials having $\mathrm{sp}^{2}$ hybridization and thus no out of plane bonds has witnessed a growing interest ${ }^{25-32}$. In particular, graphene has emerged as a promising substrate for the SAG of GaN NWs ${ }^{33-35}$ by PA-MBE. In particular, in a previous study ${ }^{33}$ we have demonstrated that GaN NWs nucleate preferentially on graphene if the latter is transferred on an amorphous substrate. Vertically aligned GaN NWs were obtained on graphene domains, with a negligible growth on the surrounding $\mathrm{SiO}_{2}$ surface. It has also been shown that the NWs exhibit the same in-plane orientation on the areas corresponding to monocrystalline grains of graphene. Based on this observation, an epitaxial relationship between graphene and GaN was proposed with a supercell alignment yielding a lattice mismatch of $3.1 \%{ }^{33}$. These results show that graphene can be used as a seed layer for the selective epitaxy of GaN NWs. III-N NWs grown on graphene can be particularly interesting for the fabrication of flexible devices ${ }^{36-39}$. Indeed, the weak interaction between graphene and its carrier substrate must facilitate the mechanical peel off a NW array and subsequent transfer to a different host substrate, such as metal or plastic. Also, GaN NWs grown on chemical-vapour deposited (CVD)-graphene were shown to have excellent optical quality ${ }^{40}$.

In all previous studies, the NW growth was performed on either large areas of graphene or on self-assembled graphene domains. For many device applications, it is important to fabricate regular arrays of NWs at predefined positions. To this end, the present work investigates the SAG of GaN NWs on CVD-graphene nanopatches defined by lithography. It is demonstrated that vertical NWs can be grown with excellent selectivity on graphene nanodots of different sizes. The photoluminescence linewidth of the resulting NWs is similar to the one of reference wires grown on AlN/Si substrates. A model quantitatively describing the impact of the dot size on the morphology and height uniformity of the NWs is proposed.

\section{EXPERIMENTAL SECTION}

We used commercially available CVD polycrystalline graphene (grain size up to $20 \mu \mathrm{m}$ ). Patches of about $1 \mathrm{~cm}^{2}$ were wet-transferred from their carrying copper foils to $1 / 41$ inch $\mathrm{Si}(100)$ wafers covered by $300 \mathrm{~nm}$ of thermal $\mathrm{SiO}_{2}$. The transfer procedure is described in $\mathrm{Li}$ et al. ${ }^{41}$. To pattern the transferred graphene, it was spin-coated with a ma-N negative resist, exposed to the electronbeam (e-beam), and etched with a $\mathrm{O}_{2}$ plasma. Finally, the reticulated ma-N resist on the graphene 
dots was removed in acetone and isopropanol bath. The mask design consisted of 12 sections with hexagonal-shape dots of radii between $50 \mathrm{~nm}$ and $250 \mathrm{~nm}$ arranged in a hexagonal lattice. In the same section, 5 different zones characterized by different pitches (between $0.25 \mu \mathrm{m}$ and 5 $\mu \mathrm{m})$ were defined. Several micrometers sized markers with a L-shape and the label of the reference dot radius were also lithographed in each section, to locate the patterns and to investigate the growth of the NWs on large area graphene reference. The substrates were then transferred in the PA-MBE system and heated for 5 minutes at $\sim 820{ }^{\circ} \mathrm{C}$ for outgassing. The growth of GaN NWs was carried out using a fixed V/III ratio of 1.1 and a Ga flux corresponding to an equivalent GaN planar growth rate of 0.62 monolayer (ML) per second. It has been previously shown that the growth selectivity critically depends on the substrate temperature ${ }^{40}$. Therefore, the growth temperature for all the samples was fixed at the optimized value of $815^{\circ} \mathrm{C}$.

\section{RESULTS AND DISCUSSION}

Fig. 1 shows scanning electron microscopy (SEM) images of GaN NWs obtained after 3 hours of total deposition time on $\mu \mathrm{m}$-sized markers (Fig. 1a), and on nanodots of 100 and $50 \mathrm{~nm}$ radii with a pitch of $2 \mu \mathrm{m}$ (Figs. 1b and 1c). Large markers and nanopatches both yield a perfect selectivity - vertical GaN NWs are only observed within the patterned areas, with no material deposit on the surrounding $\mathrm{SiO}_{2}$ surface. For $100 \mathrm{~nm}$ radius graphene dots (Fig. 1b), the hexagonal pattern can easily be seen. In most of these dots, multiple NWs have grown. When the dot radius $\left(R_{\text {dot }}\right)$ is reduced to $50 \mathrm{~nm}$ (Fig. 1c), single vertical NWs per dot are observed in most cases. Only $\sim 5 \%$ of the dots have 2 or $3 \mathrm{NWs}$, while $\sim 30 \%$ of the dots are empty (these positions are marked with arrows in Fig. 1c).

Similar results in terms of NW verticality and growth selectivity are obtained for the sample grown during 4 hours (Fig. 2), but the average number of NWs per dot has increased significantly with this longer growth duration. In particular, no empty sites are detected for the $50 \mathrm{~nm}$ dot radius array (Fig. 2j), and multiple NWs are present for any dot size, with a high degree of coalescence (Figs. 2b, 2e, 2h and 2k). The patterns contain some defects: line-shaped regions are visible and consist of parasitic NWs which grow vertically (Figs. 2a, 2d, 2g and 2j). We speculate that they originate from incomplete plasma removal of graphene during the 
patterning process. The parasitic growth may occur in positions where graphene forms wrinkles during the transfer.

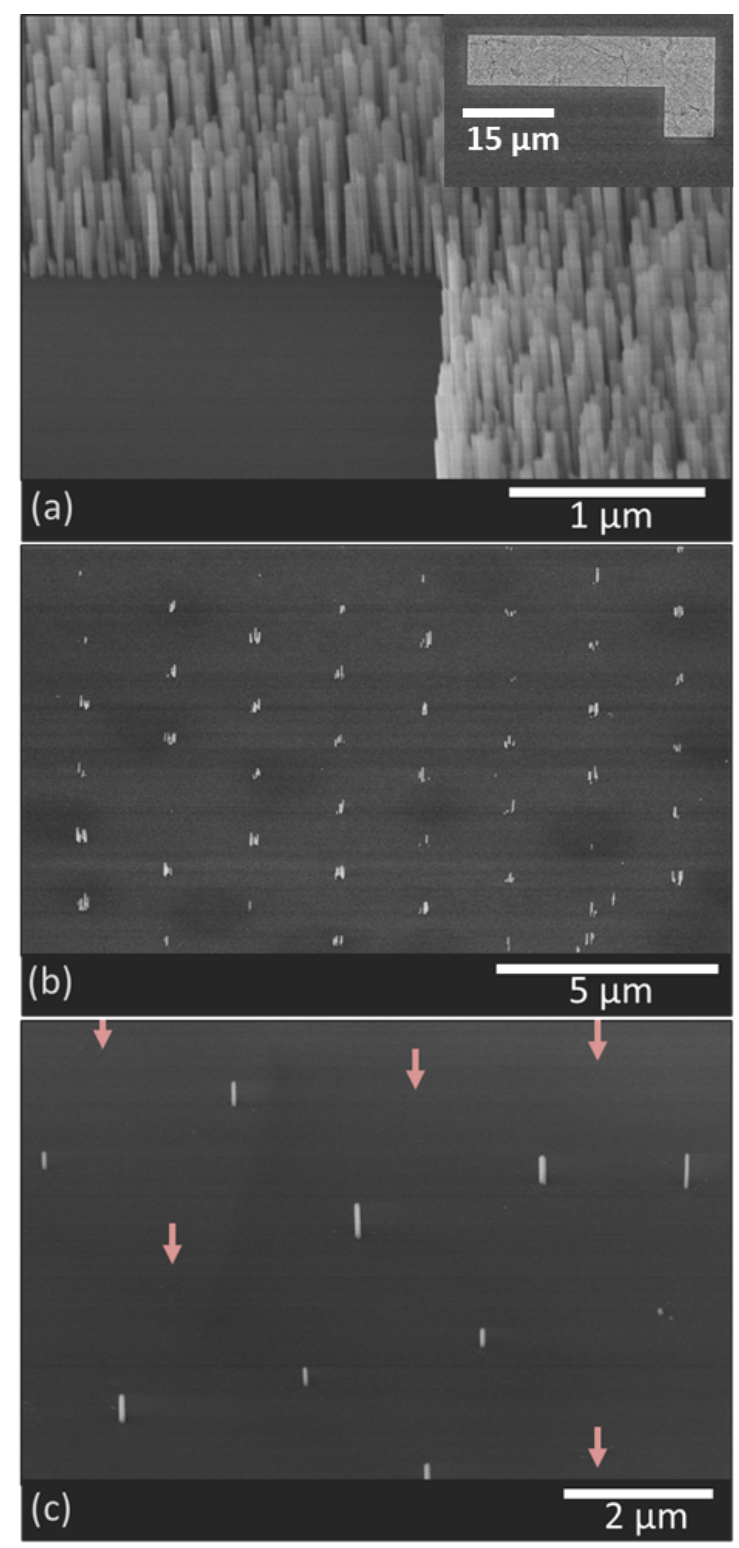

Figure 1 (a-c) SEM images of GaN NWs grown for 3 hours on (a) $\mu$ m-size patterned graphene markers, and hexagonal dots of (b) 100 and (c) $50 \mathrm{~nm}$ radius. All images are $45^{\circ}$ tilted views, except the inset to panel (a), which shows a top view. 

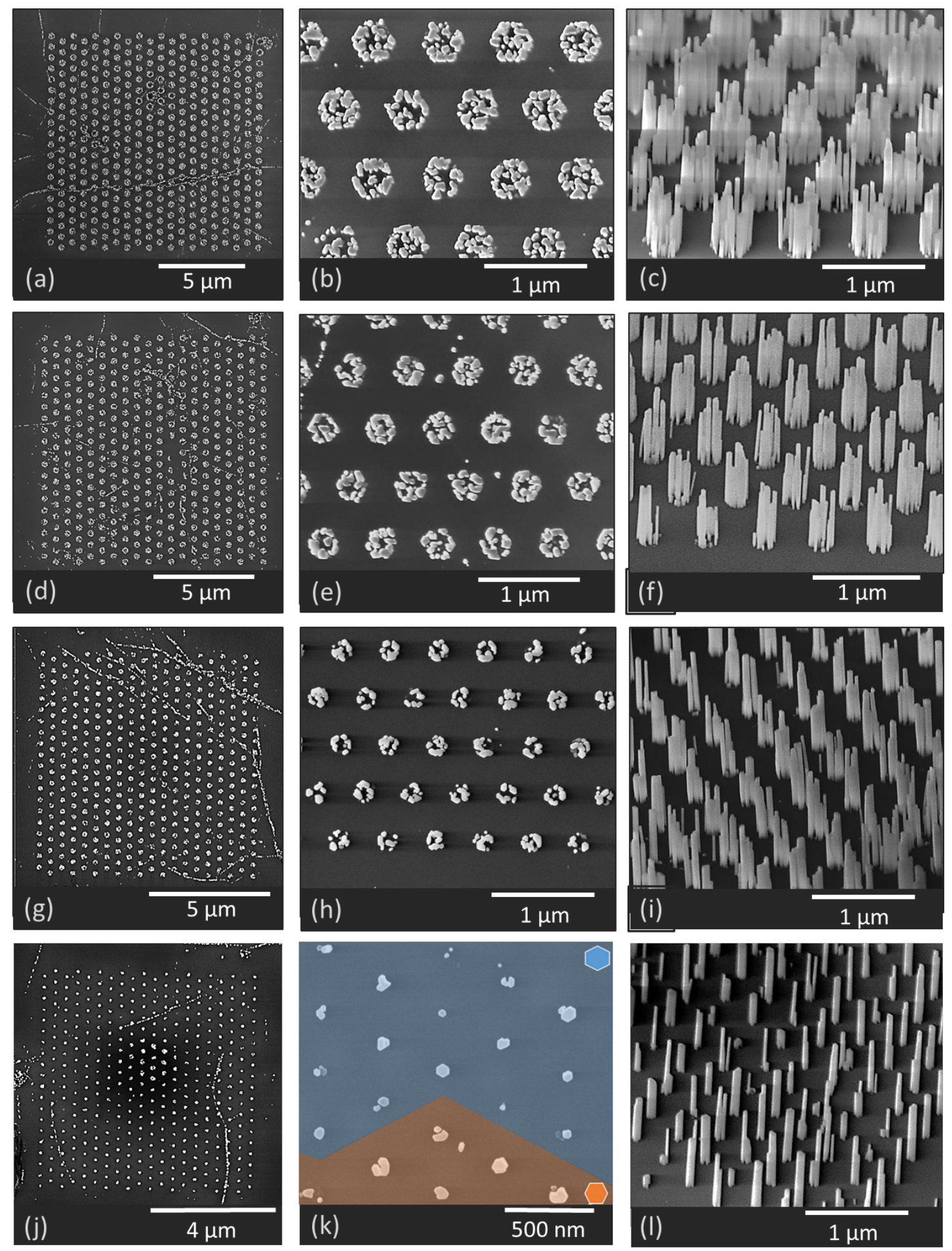

Figure 2 (a), (b), (d), (e), (g), (h), (j), (k) Top-view and (c), (f), (i), (l) $45^{\circ}$ tilted SEM images of GaN NWs grown for 4 hours on patterned graphene. The rows correspond to dot radii $R_{\text {dot }}=200$ $\mathrm{nm}$ in (a) to (c), $150 \mathrm{~nm}$ in (d) to (f), $100 \mathrm{~nm}$ in (g) to (i), and $50 \mathrm{~nm}$ in (j) to (l). 
Photoluminescence (PL) of the NWs was analysed at $5 \mathrm{~K}$ using a micro-PL set-up which consists of a continuous wave $244 \mathrm{~nm} \mathrm{Ar}{ }^{++}$laser with a spot size of around $1 \mu \mathrm{m}$, a continuous flow $\mathrm{He}$ cryostat, a UV-objective and a spectrometer equipped with a silicon charge-coupled device. Fig. 3 displays the micro-PL spectra acquired from a NW ensemble grown on a reference $\mathrm{AlN} / \mathrm{Si}(111)$ substrate and on a nano-patterned graphene on $\mathrm{SiO}_{2}$ substrate (dot diameter 250 $\mathrm{nm})$. Both samples show a luminescence dominated by the donor-bound exciton recombination $\left(\mathrm{D}^{0}, \mathrm{X}_{\mathrm{A}}\right)$, peaked around $3.471 \mathrm{eV}$. The full width at half maximum of the main peak is $1.2 \mathrm{meV}$ for both samples. Contributions from the free exciton recombination, free exciton A transition $\left(\mathrm{F}, \mathrm{X}_{\mathrm{A}}\right)$ at $3.478 \mathrm{eV}$ (for both systems) and donor bound exciton recombination $3.475 \mathrm{eV}\left(\mathrm{D}^{0}, \mathrm{X}_{\mathrm{B}}\right)$ are also observed on the high energy part of the main peak. The observed similarity of the optical features of GaN NWs grown on nano-pattered graphene and on a reference bulk AlN/Si(111) substrate confirms that the use of the nano-pattered graphene does not degrade the optical quality of the NWs. Moreover, the emission at $3.45 \mathrm{eV}$ characteristic of inversion domain boundaries ${ }^{42}$ is pronounced in GaN NWs on AlN/Si substrate, but is not observed on nano-patterned graphene as has been previously reported for graphene domains ${ }^{40}$. We speculate that the formation of inversion domain boundaries is related with the NW nucleation step and it is less likely to occur when the nucleation is slowed down. 


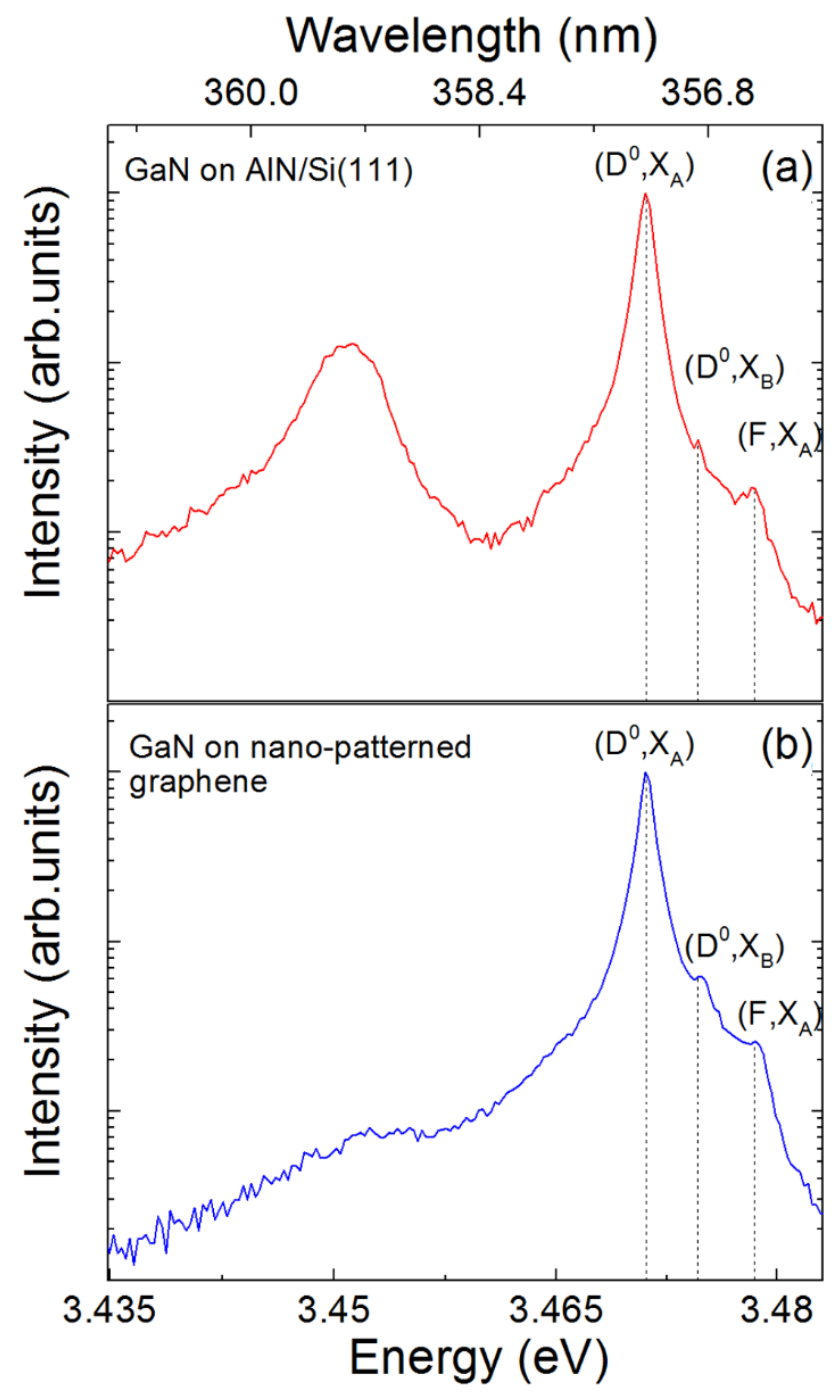

Figure 3 Low temperature micro-PL spectra of GaN NWs grown on (a) AlN/Si(111) and (b) nano-patterned graphene on $\mathrm{SiO}_{2} / \mathrm{Si}(100)$ substrates.

We now study in more detail how the number of NWs per graphene dot evolves with the growth time. For the 3 hours sample, single NW occupation is obtained for the smallest dots which are, nevertheless, larger than the NW footprint $\left(R_{\text {dot }}=50 \mathrm{~nm}\right.$, compared to a typical NW radius of 20 $\mathrm{nm})$. For larger dots with multiple NWs, the graphene surface is not covered uniformly: the NWs nucleate preferentially at the dot periphery (see Fig. 4a). The same tendency is observed in the 4 hours sample (Fig. 4b). In this case, the NW density is higher at the edges, leading to some NWs coalescence, and the inner dot surface is partially filled with NWs. This phenomenon is similar to the findings of Ishizawa and co-workers ${ }^{14}$ on nitridated $\mathrm{Al}$ nanodots, where the nucleation of 
NWs was found to be peripheral. The preferential NW formation at the dots periphery leads to a linear increase of the number of nucleated NWs per graphene dot with the dot size (plotted for the 4 hours growth sample in Fig. 4c).
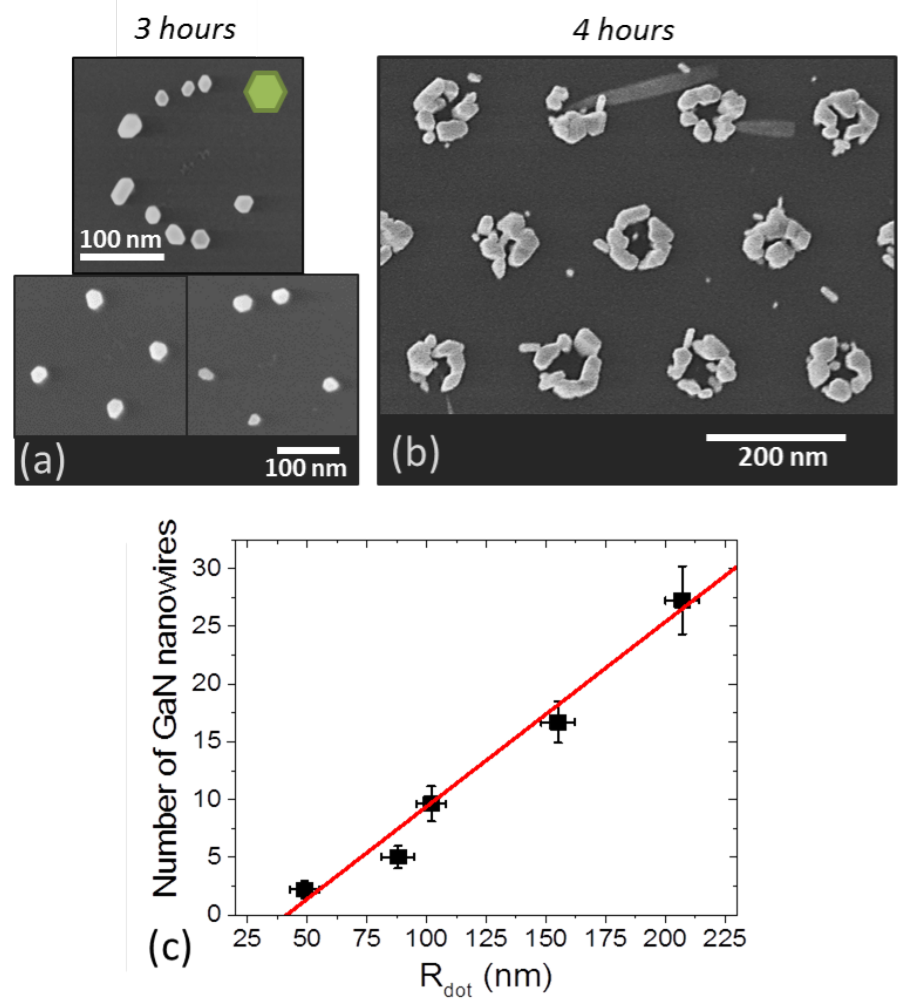

Figure 4 Top-view SEM images of GaN NWs grown on patterns with $\mathrm{R}_{\mathrm{dot}}=100 \mathrm{~nm}$ for (a) 3 and (b) 4 hours. The green hexagon in (a) shows the orientation of the NW m-planes. (c) Variation of the number of NWs per graphene dot as a function of $\mathrm{R}_{\mathrm{dot}}$ ( 4 hours growth sample).

Within each graphene dot, the vertical facets of the NWs indicate that they all have the same basal orientation (Fig. 4a), and the in-plane coherence is preserved also among neighboring dots, over areas of several tens of $\mu \mathrm{m}^{2}$ (Fig. 2k). This is consistent with the typical size of monocrystalline grains in the transferred CVD-graphene (surface grain area $\sim 20 \mu \mathrm{m}^{2}$ ), and confirms that the NWs establish an epitaxial alignment with graphene. Indeed, a random in-plane orientation is expected for NWs growing on an amorphous substrate like $\mathrm{SiO}_{2}^{43}$.

All these observations strongly support the idea that within our experimental conditions, the crystalline structure of graphene is sufficiently preserved after the lithography, etching and growth steps. However, the preferential nucleation of NWs on the periphery also suggests that local deviations from the ideal (pristine) configuration are favorable nucleation sites. In fact, in 
comparison to as-grown graphene domains ${ }^{33}$, graphene dots defined by e-beam lithography and plasma etching are prone to contain carbon dangling bonds at their edges or edge reconstructions ${ }^{44}$. Theoretically, these defects can enhance nitrogen incorporation from nitrogen plasma or even Ga incorporation ${ }^{45,46}$, favoring in turn $\mathrm{NW}$ nucleation. More detailed investigations, focused on the study of the GaN nucleation and GaN/graphene interface are needed to elucidate these points.

On different arrays for both 3 hours and 4 hours of growth, the average NW morphology does not depend on the graphene dot pitch, but shows an evident dependency from the graphene dot size. Fig. 5 shows the results of a statistical analysis performed on the sample grown for 4 hours (arrays of $250 \mathrm{~nm}$ pitch). For each dot size, the length of about $100 \mathrm{NWs}$ was measured to obtain the distributions shown in Fig. 5a and summarized in the plot of Fig. 5b. When $\mathrm{R}_{\text {dot }}$ increases from 50 to $100 \mathrm{~nm}$, the mean NW length increases significantly, from $420 \pm 100 \mathrm{~nm}$ to $580 \pm 100$ $\mathrm{nm}$ (Fig. 5b). For larger dots, the length stabilizes at $640 \pm 110 \mathrm{~nm}$. This upper value equals the average NW length measured on the large L-shape reference area $(630 \pm 100 \mathrm{~nm})$.

Under N-rich conditions the axial growth rate of non-tapered NWs is limited by the Ga supply to the NW top ${ }^{47}$. This may include the direct impingement and the surface diffusion along the sidewalls. The diffusion length of Ga adatoms at $815^{\circ} \mathrm{C}$ should be very short, of about $40 \mathrm{~nm}^{48}$. Since the NW lengths are one order of magnitude larger than this value, much shorter NWs on smaller graphene dots cannot be explained solely by their slower axial growth rate originating from a lower collection of adatoms diffusing from the underlying graphene dot. We note that the length distributions present a pronounced asymmetry: a long tail is observed towards short lengths. Assuming a constant NW elongation rate, this tail corresponds to the NWs that have emerged lately during the growth process. This phenomenon can be associated to the long incubation time of GaN NWs on graphene ${ }^{40}$. Furthermore, the length distribution for $\mathrm{R}_{\mathrm{dot}}=50$ $\mathrm{nm}$ does not tend to zero at zero length: this indicates that after 4 hours of growth, the nucleation of new NWs is still possible on these small dots. It is possible that on graphene dots smaller than a certain critical size, GaN nucleation could become even more difficult, resulting in an incomplete nucleation and shorter effective growth time, and consequently in a smaller NW length. 

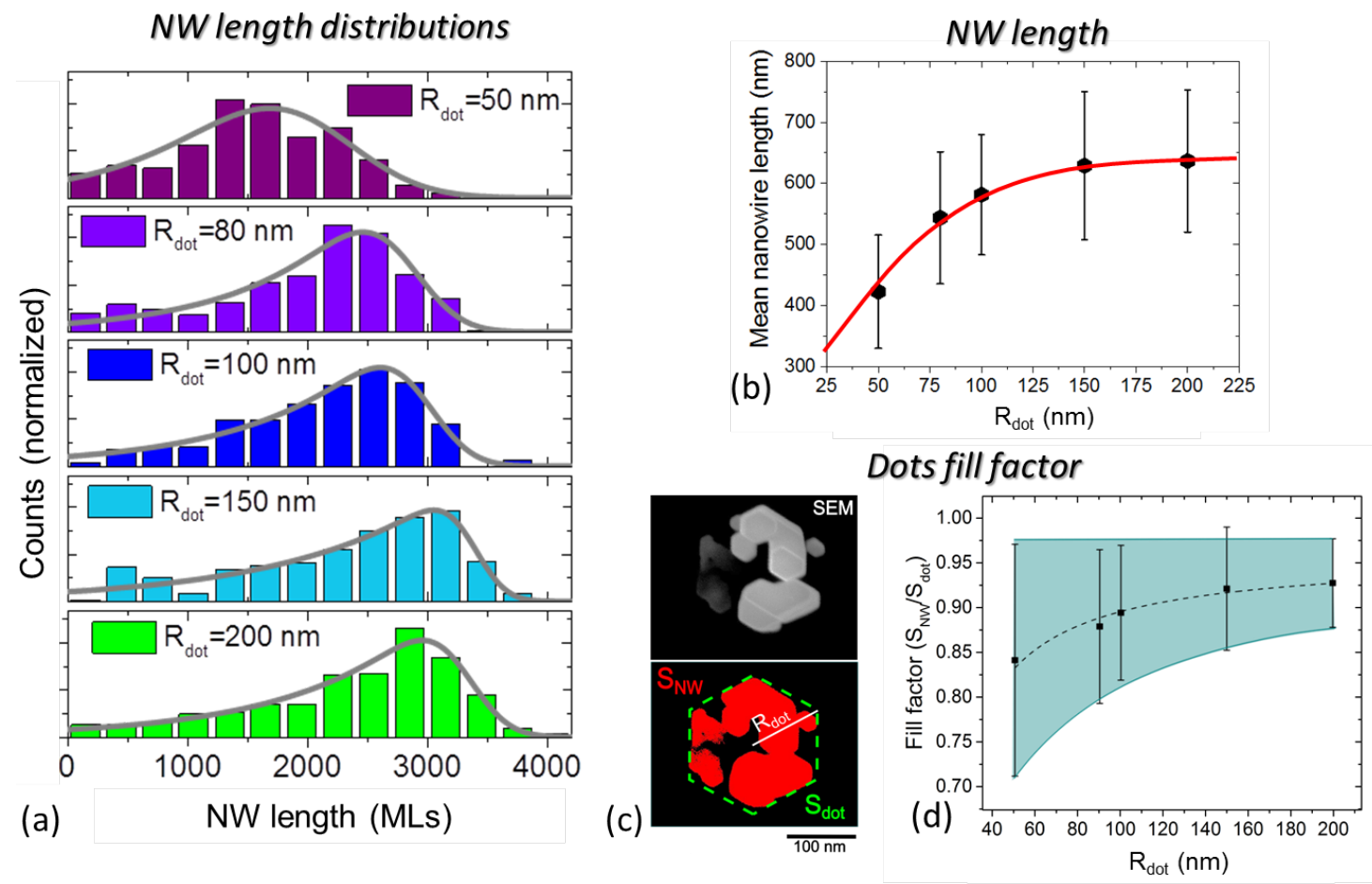

Figure 5 (a) Length distributions of GaN NWs for different radii of graphene dots (histograms), fitted by the model equation (full lines). (b) Average NW length as a function of the dot radius. The line shows the fit by the model described in the text. (c) SEM image (top) and estimation of the surface area occupied by NWs using IMAGEJ software, on a $100 \mathrm{~nm}$ radius graphene dot. (d) Variation of the average filling factor $S_{\mathrm{NW}} / S_{\text {dot }}$ versus $R_{\text {dot }}$. Dashed line is guide for the eye. Blue stripe marks the increasing standard deviation for smaller dot radii.

To investigate this point, the filling factor of the dots, defined as the fraction of the dot area occupied by NWs, is an informative parameter to analyze. Using top-view SEM images, the edges of the NWs (or of coalesced clusters of NWs) were detected and the graphene surface area covered by NWs, $\mathrm{S}_{\mathrm{NW}}$, was estimated (Fig. 5c). In each array, this analysis was repeated for 30 individual graphene dots of same nominal area $S_{\text {dot }}$. In Fig. $5 d$ we see that the filling factor, $\mathrm{S}_{\mathrm{NW}} / \mathrm{S}_{\mathrm{dot}}$, increases from 0.84 to about 0.92 as the dot radius increases from 50 to $150 \mathrm{~nm}$, and then saturates at about 0.93 . In parallel, the filling factor standard deviation decreases from about $15 \%$ to about $5 \%$. As a first approximation, we can consider $\mathrm{S}_{\mathrm{NW}} / \mathrm{S}_{\mathrm{dot}}$ to be proportional to the NW number per surface unit, and its saturation value to the NW density leading to a fluxes shadowing ${ }^{49}$ of the graphene surface (so that no nucleation is possible anymore). Its lower value for the smaller dots suggests again that the NW nucleation stage is not fully completed at the end of the growth process for these dots. Also, the higher fill factor standard deviation indicates a 
wider spread in time of the nucleation events. Both these evidences are consistent with an increased NW incubation time (i.e., by a delayed nucleation of individual NWs) on the smaller dots.

Based on these considerations, we now present a model to quantitatively describe our experimental data. The NW length distribution is modeled under the following assumptions. The entire growth process is divided into two stages: (i) the incubation stage, lasting over the period of time $\tau_{\mathrm{i}}$ after the beginning of GaN deposition, at the end of which the first NW seed forms; (ii) the elongation stage, where the NWs grow axially at a constant growth rate $v$. We thus neglect possible shape transitions of NWs at the beginning of growth ${ }^{50}$, and a short stage after the NW nucleation in which the diffusion flux of Ga depends on the NW length ${ }^{51,52}$.

Taking these features in consideration, we describe the NW length distribution in the form ${ }^{53}$

$$
f(l, t)=\frac{\alpha}{2 A} e^{\left(\alpha\left(l-\tau+\tau_{i}\right)+\frac{\alpha^{2} \sigma_{0}^{2}}{2}\right)} \operatorname{erfc}\left(\frac{\alpha \sigma_{0}^{2}+l+\tau+\tau_{i}}{\sqrt{2 \sigma_{0}}}\right)
$$

Here, $l$ is the NW length (measured in numbers of MLs of GaN along the c-axis), $\tau=v \cdot t$ is the reference NW length (the mean length which would have been obtained without any nucleation delay as introduced in Ref [52]), and $t$ is time. The $\sigma_{0}$ parameter describes the standard deviation (with $\sigma_{0}^{2}$ as the corresponding variance) of Green's function ${ }^{53}$, that is, the intrinsic length distribution width for the same ensemble of NWs without the nucleation delay. The parameter $\alpha<1$ gives the measure of this delay. The $\operatorname{erfc}(y)$ is the regularized complimentary error function, defined as

$$
\operatorname{erfc}(y)=\frac{2}{\sqrt{\pi}} \int_{y}^{\infty} d t e^{-t^{2}}
$$

Finally, $A=\int_{0}^{\infty} d s f(s, \tau)$ is the normalization constant accounting for the fact that some nucleation sites may not be occupied at the moment of the growth stop. Overall, the shape of this length distribution is controlled by the three parameters, $\tau-\tau_{i}, \sigma_{0}$ and $\alpha$. We will see that is close to unity for all our NWs, in which case the mean NW length and the length distribution variance are given by $\left\langle l>=\tau-\tau_{i}-1 / \alpha\right.$ and $\sigma^{2}=\sigma_{0}^{2}+1 / \alpha^{2}$, respectively. It should be noted that the length distribution given by Eq. (1) and the corresponding parameters refer to the ensembles of individual non-interacting NWs and do not take into account their possible coalescence in a later growth stage. 
Fig. 5(b) shows the best fits of the experimental length distributions by Eq. (1) with the parameters summarized in Table 1. As mentioned earlier, the length distributions are asymmetric, and the wide tail on the shorter NW length side reflects the presence of NWs that have nucleated lately. This is quantified by the dimensionless parameter $\alpha$, which is of the order of $10^{-3}$. It means that the formation of the very first NW ML takes approximately 1000 times longer than the formation of one ML during the steady-state elongation. As expected, the extracted values of the incubation time show a strong dependence on the dot size. As illustrated in Fig. 6, $\tau_{i}$ tends to an asymptote for $\mathrm{R}_{\mathrm{dot}}$ above $200 \mathrm{~nm}$, while it increases exponentially for smaller dots. By assuming that the asymptotic value of incubation time for big dot radii is of 1 hour 20 minutes (which is the typical value measured by RHEED on $1 \mathrm{~cm}^{2}$ graphene patches under identical growth conditions ${ }^{40}$ ), we estimate that for a $50 \mathrm{~nm}$ dot radius, $\tau_{\mathrm{i}}$ should increase to about 2 hours. Note that with other conditions and other substrates, long incubation times have been already reported for GaN NW growth ${ }^{52,54}$. Broad length distributions caused by difficult NW nucleation has been seen not only for III-V NWs grown by the vapor-liquid-solid mechanism on unpatterned substrates ${ }^{53}$, but also in regular arrays on lithographically patterned $\mathrm{SiO}_{\mathrm{x}} / \mathrm{Si}(111)$ surfaces $^{55}$. Quite clearly, our SAG growth of GaN NWs on graphene dots proceeds at much lower growth rate, so the observed spread of the length distributions is not surprising.

Table 1. Experimental and fitting parameters of GaN NW length distributions.

\begin{tabular}{|c|c|c|c|c|c|c|}
\hline $\begin{array}{c}\text { Radius of } \\
\text { graphene } \\
\text { dot }(\mathrm{nm})\end{array}$ & $\begin{array}{c}\text { Measured } \\
\text { mean } \\
\text { length }<>> \\
(\mathrm{MLs})\end{array}$ & $\begin{array}{c}\text { Measured } \\
\text { standard } \\
\text { deviation } \\
(\mathrm{MLs})\end{array}$ & $\begin{array}{c}\tau-\tau_{i} \\
(\mathrm{MLs})\end{array}$ & $\begin{array}{c}\text { Intrinsic } \\
\text { distribution } \\
\text { width } \\
\sigma_{0} \\
(\mathrm{MLs})\end{array}$ & $\begin{array}{c}\text { Nucleation } \\
\text { delay } \\
\alpha\end{array}$ & $A$ \\
\hline 50 & 1565 & 687 & $2156 \pm 114$ & $542 \pm 109$ & $0.0013 \pm 0.0001$ & 0.89 \\
\hline 80 & 2013 & 800 & $2842 \pm 52$ & $319 \pm 63$ & $0.0011 \pm 0.0001$ & 0.97 \\
\hline 100 & 2153 & 729 & $2982 \pm 27$ & $288 \pm 35$ & $0.0010 \pm 0.0001$ & 0.95 \\
\hline 150 & 2329 & 898 & $3380 \pm 38$ & $209 \pm 53$ & $0.0008 \pm 0.0001$ & 0.96 \\
\hline 200 & 2358 & 861 & $3320 \pm 36$ & $268 \pm 46$ & $0.0009 \pm 0.0001$ & 0.96 \\
\hline
\end{tabular}




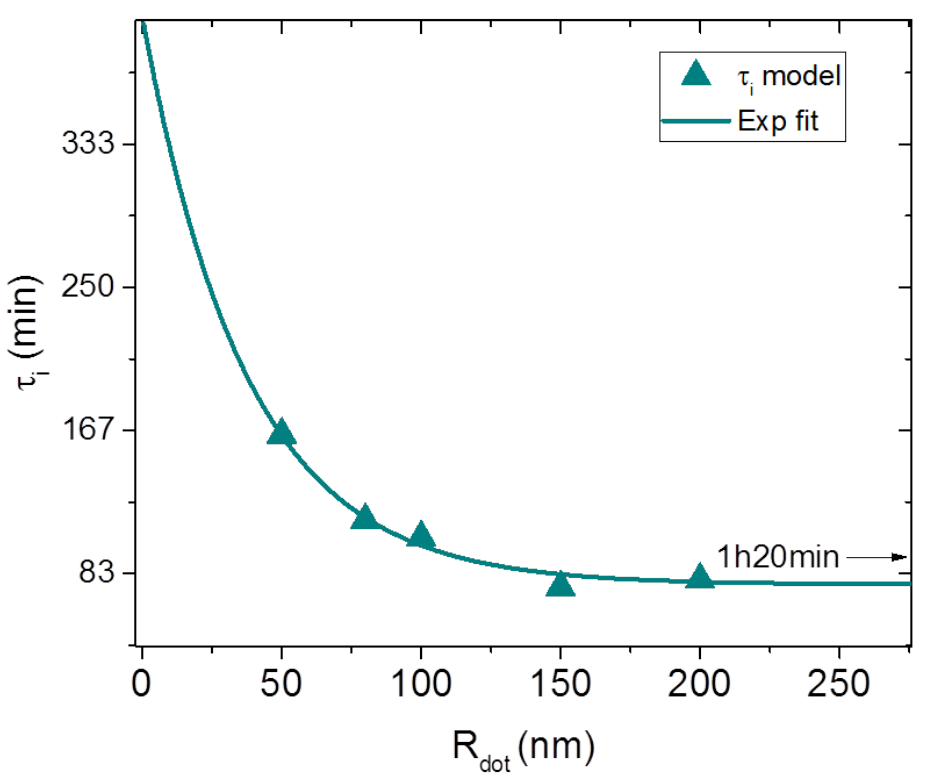

Figure 6 Incubation time $\tau_{i}$ as a function of $\mathrm{R}_{\text {dot. }}$ Line shows the exponential fit.

We now consider the origin of the incubation time dependence on graphene dot size. We first recall that the number of NWs per dot increases almost linearly with $R_{\text {dot }}$ (Fig. 4c). This reflects the preferential nucleation at the graphene dot periphery where the number of possible nucleation sites is proportional to $\mathrm{R}_{\mathrm{dot}}$. Hence, the area of one nucleation site in the $\operatorname{dot} \pi R_{d o t}^{2} / N$ is roughly proportional to $\mathrm{R}_{\mathrm{dot}}$. We assume that the NW nucleation is limited by the Ga desorption. In fact, other experiments confirmed a faster NW nucleation for growths carried out with higher Ga fluxes, as is usually observed on more conventional crystalline substrates ${ }^{52,54,56}$. Assuming that Ga atoms diffuse on graphene and their mean path before desorption is limited by the diffusion length $\lambda_{G a}$, the effective Ga flux per nucleation site $\Phi$ equals the total flux divided to the area of the site, that is $c_{1} / R_{d o t}$, when $R_{d o t}<<\lambda_{G a}$, with a positive constant $c_{l}$. For larger dots with $R_{d o t}>>\lambda_{G a}$, the Ga flux per site equals the total flux divided to the diffusion area, that is, $c_{2} /$ $\lambda_{G a}^{2}$ with a positive constant $c_{2}$. These expressions show that for smaller dots, there is a competition between the neighboring nucleation sites for the arriving $\mathrm{Ga}$, whereas for larger dots the flux per site is limited by the $\mathrm{Ga}$ diffusion length and becomes size-independent. The incubation time is inversely proportional to $\Phi$ (Ref. [54]) and hence proportional to $R_{\text {dot }}$ for $R_{d o t}<<\lambda_{G a}$ and independent of $R_{\mathrm{dot}}$ for $R_{d o t}>>\lambda_{G a}$. For a time-independent axial NW growth rate, the simplest fitting formula for the mean NW length, which is reduced to these two asymptotes for small and large graphene dots, can be put as 


$$
<l>=<l>_{\max }\left(1-e^{-\frac{R_{d o t}}{\lambda_{G a}}}\right) ;
$$

where $\langle l\rangle_{\max }$ is the asymptotic NW length at $R_{d o t} \gg \lambda_{G a}$. Fitting the measured average NW length dependency on the graphene dot radius (Fig. 5(b)) with this simple model yields a plausible Ga diffusion length of $45 \mathrm{~nm}$.

In this model, we neglected a possible contribution of $\mathrm{Ga}$ adatoms diffusing from the mask onto the graphene dot. This choice can be justified as follows. First, no differences in the NW length and filling factor are observed for the different inter-dot pitches (varied from $5 \mu \mathrm{m}$ down to 250 $\mathrm{nm}$ ). Second, for growths carried out at lower temperature (such that GaN NWs start to grow also on $\mathrm{SiO}_{2}$ ), there is no clear evidence of a depletion region in the vicinity of graphene edges. Hence, it is reasonable to assume that the current of $\mathrm{Ga}$ from the $\mathrm{SiO}_{2}$ surface to the dot is very small compared to the one generated by the direct impingement. This should be due to a very small diffusion length of $\mathrm{Ga}$ on $\mathrm{SiO}_{2}$ at these temperatures, and/or to a high diffusion barrier at the graphene $/ \mathrm{SiO}_{2}$ edge.

Overall, the presented model correctly describes the entire set of experimental data. It describes the asymmetric NW length distributions and the reduction of the NW length on small graphene dots caused by difficult nucleation in the confined graphene areas. We note that the increase of the incubation time and of the intrinsic length distribution width for small $\mathrm{R}_{\mathrm{dot}}$ can be a drawback for device applications where the length homogeneity is required. A two-step growth protocol with a first step accelerating the NW nucleation and the second step favoring high selectivity can solve this issue. This idea has been originally developed by Carnevale et al.$^{57}$, with the difference that in the case of graphene it seems more suitable to play with the Ga flux rather than with the growth temperature. In preliminary growth experiments on large-area graphene patches, we have observed a two-fold reduction of the nucleation time by increasing the Ga flux at the initial growth stage. A further refinement of this strategy applied to nano-patterned graphene is expected to enable the growth of homogeneous NW arrays with $1 \mathrm{NW}$ per graphene dot.

\section{CONCLUSIONS}

We have demonstrated a novel SAG strategy for GaN NWs using graphene nano-dots. The growth was performed by PA-MBE on graphene transferred on $\mathrm{SiO}_{2}$ and patterned by e-beam

lithography. Vertical and in-plane oriented ensembles of NWs were obtained on dots of different 
sizes. We have shown that nucleation takes place preferentially at the borders of the graphene dots, resulting in a linear dependence of the NW number versus dot radius. The asymmetric NW length distributions were correctly described by a model which takes into account a dot-sizedependent incubation time. We deduce that this incubation time increases significantly for dots radii lower than $150 \mathrm{~nm}$, which results in a decreased mean NW length, in a broader length distribution and in a reduced NW filling factor. The proposed SAG approach combined with the easy NW peel-off enabled by the weak van der Waals adhesion of graphene on its carrier substrate, opens new fabrication routes for mechanically flexible NW devices. Further efforts are required, however, to minimize the effects of polynucleation and coalescence on the structural and optical properties of SAG GaN NWs on patterned graphene.

\section{AUTHOR INFORMATION}

Corresponding Author

Martina.Morassi@u-psud.fr

Maria.Tchernycheva@u-psud.fr

ORCID:

Andrey Babichev: A.0000-0002-3463-4744

Maria Tchernycheva : 0000-0003-4144-0793

\section{Notes}

The authors declare no competing financial interest.

\section{AKNOWLEDGEMENTS}

The authors acknowledge the financial support from EU ERC project "NanoHarvest" (grant no. 639052), the French "Agence Nationale de la Recherche" (ANR) through "GaNeX" (ANR-11LABX-2014) and "NanoSaclay" (ANR-10-LABX-0035) programs. AB acknowledges the support of the Russian Science Foundation (project no. 18-72-00157) for the part of graphene transfer. VGD and YB thank the Russian Science Foundation for financial support under Grant 19-72-30004.

\section{BIBLIOGRAPHY}

(1) Li, S.; Waag, A. GaN based nanorods for solid state lighting. J. Appl. Phys. 2012, 111, 071101. 
based visible light emitters. Semicond. Semimetals 2016, 94, 221-227.

(3) Morassi, M.; Largeau, L.; Oehler, F.; Song, H.-G.; Travers, L.; Julien, F. H.; Harmand, J.-C.; Cho, Y.-H.; Glas, F.; Tchernycheva, M.;Gogneau, N.. Morphology tailoring and growth mechanism of in-rich InGaN/GaN axial nanowire heterostructures by plasma-assisted molecular beam epitaxy. Cryst. Growth Des. 2018, 18, 2545-2554.

(4) Kikuchi, A.; Kawai, M.; Tada, M.; Kishino, K. InGaN/GaN multiple quantum disk nanocolumn light-emitting diodes grown on (111) Si substrate. Jpn. J. Appl. Phys. 2004, 43, L1524-L1526.

(5) Kishino, K.; Yanagihara, A.; Ikeda, K.; Yamano, K. Monolithic integration of four-colour InGaN-based nanocolumn LEDs. Electron. Lett. 2015, 51, 852-854.

(6) Limbach, F.; Hauswald, C.; Lähnemann, J.; Wölz, M.; Brandt, O.; Trampert, A.; Hanke, M.; Jahn, U.; Calarco, R.; Geelhaar, L.; et al. Current path in light emitting diodes based on nanowire ensembles. Nanotechnology 2012, 23, 465301.

(7) Bavencove, A.-L.; Tourbot, G.; Garcia, J.; Désières, Y.; Gilet, P.; Levy, F.; André, B.; Gayral, B.; Daudin, B.; Dang, L. S.

Submicrometre resolved optical characterization of green nanowire-based light emitting diodes. Nanotechnology 2011, $22,345705$.

(8) Albert, S.; Bengoechea-encabo, A.; Lefebvre, P.; Barbagini, F.; Trampert, A. Selective area growth and characterization of InGaN nano-disks implemented in GaN nanocolumns with different top morphologies. Appl. Phys. Lett. 2012, 100, 231906.

(9) Albert, S.; Bengoechea-Encabo, A. M.; Sánchez-García, M. Á.; Calleja, E. Selective area growth of InGaN/GaN nanocolumnar heterostructures by plasma-assisted molecular beam epitaxy. Semicond. Semimetals 2017, 96, 231-266.

(10) Lazić, S.; Chernysheva, E.; Gačević, Ž.; García-Lepetit, N.; van der Meulen, H. P.; Müller, M.; Bertram, F.; Veit, P.; Christen, J.; Torres-Pardo, A.; González-Calbet, J. M.; Calleja E. Ordered arrays of InGaN/GaN dot-in-a-wire nanostructures as single photon emitters. Proc. SPIE 2015, 9363, 93630U.

(11) Gačević, Ž.; Vukmirović, N.; García-Lepetit, N.; Torres-Pardo, A.; Müller, M.; Metzner, S.; Albert, S.; Bengoechea-Encabo, A.; Bertram, F.; Veit, P.; Christen, J.; González-Calbet, J. M.; Calleja E. Influence of composition, strain, and electric field anisotropy on different emission colors and recombination dynamics from ingan nanodisks in pencil-like GaN nanowires. Phys. Rev. B 2016, 93, 125436.

(12) Sekiguchi, H.; Kishino, K.; Kikuchi, A. Ti-mask selective-area growth of GaN by rf-plasma-assisted molecular-beam epitaxy for fabricating regularly arranged InGaN/GaN nanocolumns. Appl. Phys. Express 2008, 1, 124002.

(13) Kishino, K.; Ishizawa, S. Selective-area growth of gan nanocolumns on Si ( 111 ) substrates for application to nanocolumn emitters with systematic analysis of dislocation filtering effect of nanocolumns. Nanotechnology 2015, 26, 1-13.

(14) Ishizawa, S.; Kishino, K.; Kikuchi, A. Selective-area growth of GaN nanocolumns on Si(111) substrates using nitrided al nanopatterns by rf-plasma-assisted molecular-beam epitaxy. Appl. Phys. Express 2008, 1, 3-6.

(15) Schumann, T.; Gotschke, T.; Limbach, F.; Stoica, T.; Calarco, R. Selective-area catalyst-free mbe growth of GaN nanowires using a patterned oxide layer. Nanotechnology 2011, 22, 095603.

(16) Kruse, J. E.; Lymperakis, L.; Eftychis, S.; Adikimenakis, A.; Doundoulakis, G.; Tsagaraki, K.; Androulidaki, M.; Olziersky, A.; Dimitrakis, P.; Ioannou-Sougleridis, V.; Normand, P.; Koukoula, T.; Kehagias, Th.; Komninou, Ph.; Konstantinidis, G. Georgakilas A. Selectivearea growth of $\mathrm{GaN}$ nanowires on $\mathrm{SiO}_{2}$-masked $\mathrm{Si}$ (111) substrates by molecular beam epitaxy. J. Appl. Phys. 2016, 119, 224305.

(17) Bertness, B. K. A.; Sanders, A. W.; Rourke, D. M.; Harvey, T. E.; Roshko, A.; Schlager, J. B.; Sanford, N. A. Controlled nucleation of 
GaN nanowires grown with molecular beam epitaxy. Adv. Mater. 2010, 20, 2911-2915.

(18) Brubaker, M. D.; Du, S. M.; Harvey, T. E.; Blanchard, P. T.; Roshko, A.; Sanders, A. W.; Sanford, N. A.; Bertness, K. A. Polaritycontrolled GaN/AlN nucleation layers for selective-area growth of GaN nanowire arrays on $\mathrm{Si}(111)$ substrates by molecular beam epitaxy. Cryst. Growth Des. 2015, 16, 596-604.

(19) Schuster, F.; Hetzl, M.; Weiszer, S.; Garrido, J. A.; De La Mata, M.; Magen, C.; Arbiol, J.; Stutzmann, M. Position-controlled growth of GaN nanowires and nanotubes on diamond by molecular beam epitaxy. Nano Lett. 2015, 15, 1773-1779.

(20) Gacevic, Z.; Gomez Sanchez, D.; Calleja, E. Formation mechanisms of GaN nanowires grown by selective area growth homoepitaxy. Nano Lett. 2015, 15, 1117-1121.

(21) Kano, T.; Yoshida, J.; Miyagawa, R.; Mizuno, Y.; Oto, T. GaN nanocolumn arrays with diameter $<30$ nm prepared by two-step selective area growth. Electron. Lett. 2015, 51, 2125-2126.

(22) Bengoechea-encabo, A.; Barbagini, F.; Fernandez-garrido, S.; Grandal, J.; Ristic, J. Understanding the selective área growth of GaN nanocolumns by MBE using Ti nanomasks. J. Cryst. Growth 2011, 325, 89-92.

(23) Kishino, K.; Hoshino, T.; Ishizawa, S.; Kikuchi, A. Selective-area growth of GaN nanocolumns on titanium-mask-patterned silicon (111) substrates by rf-plasma-assisted molecular-beam epitaxy. Electron. Lett. 2008, 44, 819.

(24) Sanford, N. A.; Blanchard, P. T.; Brubaker, M.; Bertness, K. A.; Roshko, A.; Schlager, J. B.; Kirchhofer, R.; Diercks, D. R.; Gorman,

B. Laser-assisted atom probe tomography of MBE grown GaN nanowire heterostructures. Phys. Status Solidi 2014, 11, 608-612.

(25) Heilmann, M; Munshi, AM; Sarau, G; Göbelt, M; Tessarek, C; Fauske, V. T., van Helvoort, A. T. J.; Yang, J.; Latzel, M.; Hoffmann, B.; Conibeer, G.; Weman, H.; Christiansen, S. Vertically oriented growth of gan nanorods on Si using graphene as an atomically thin buffer layer. Nano Lett. 2016, 16, 3524-3532.

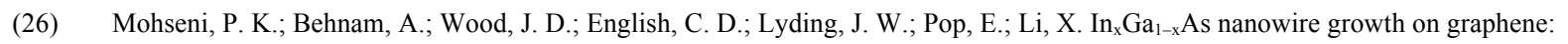
van der Waals epitaxy induced phase segregation. Nano Lett. 2013, 13, 1153-1161.

(27) Hong, Y. J.; Lee, W. H.; Wu, Y.; Ruoff, R. S.; Fukui, T. van der Waals epitaxy of InAs nanowires vertically aligned on single-layer graphene. Nano Lett. 2012, 12, 1431-1446.

(28) Munshi, A. M; Dheeraj, D. L.; Fauske, V. T.; Kim, D.-C.; van Helvoort, A. T. J.; Fimland, B.-O.; Weman, H. Vertically aligned GaAs nanowires on graphite and few-layer graphene: generic model and epitaxial growth. Nano Lett. 2012, 12, 4570-4576.

(29) Utama, M. I. B.; Belarre, F. J., Magen, C.; Peng, B.; Arbiol, J.; Xiong, Q. Incommensurate van der Waals epitaxy of nanowire arrays: a case study with $\mathrm{ZnO}$ on muscovite mica substrates. Nano Lett. 2012, 12, 2146-2152.

(30) Baxter, J. B.; Aydil, E. S. Epitaxial growth of ZnO nanowires on a- and c-plane sapphire. J. Cryst. Growth 2005, $274,407-411$.

(31) Narayan, J.; Larson, B. C.; Domain epitaxy: A unified paradigm for thin film growth. J. Appl. Phys. 2003, 93, 278-285.

(32) Ayers, J. E., Heteroepitaxy of Semiconductors: Theory, Growth, and Characterization; CRC Press: Boca Raton, FL, 2007.

(33) Kumaresan, V.; Largeau, L.; Madouri, A.; Glas, F.; Zhang, H.; Oehler, F.; Cavanna, A.; Babichev, A.; Travers, L.; Gogneau, N.;

Tchernycheva, M.; Harmand J.-C. Epitaxy of GaN nanowires on graphene. Nano Lett. 2016, 16, 4895-4902.

(34) Ramsteiner, M.; Gao, G.; Galves, L. A.; Sharma, B.; Corfdir, P.; Calabrese, G.; Schiaber, Z. D. S.; Pfu, C.; Trampert, A.; Lopes, J. M. J.; Brandt, O.; Geelhaar, L. Molecular beam epitaxy of GaN nanowires on epitaxial graphene. Nano Lett. 2017, 17, 5213-5221.

(35) Hong, Y. J.; Lee, C. H. Van der Waals heteroepitaxy of semiconductor nanowires. Semicond. Semimetals 2015, 93, $125-172$. 
(36) Lee, C. H.; Kim, Y. J.; Hong, Y. J.; Jeon, S. R.; Bae, S.; Hong, B. H.; Yi, G. C. Flexible inorganic nanostructure light-emitting diodes fabricated on graphene films. Adv. Mater. 2011, 23, 4614-4619.

(37) Guan, N.; Dai, X.; Babichev, A. V.; Julien, F. H.; Tchernycheva, M. Flexible inorganic light emitting diodes based on semiconductor nanowires. Chem. Sci. 2017, 8, 7904-7911.

(38) Chung, K.; Beak, H.; Tchoe, Y.; Oh, H.; Yoo, H.; Kim, M.; Yi, G. C. Growth and characterizations of GaN micro-rods on graphene films for flexible light emitting diodes. APL Mater. 2014, 2 (9), 092512.

(39) Chung, K.; Yoo, H.; Hyun, J. K.; Oh, H.; Tchoe, Y.; Lee, K.; Baek, H.; Kim, M.; Yi, G. Flexible GaN light-emitting diodes using gan microdisks epitaxial laterally overgrown on graphene dots. Adv. Mater. 2016, 28, 7688-7694.

(40) Mancini, L.; Morassi, M.; Sinito, C.; Brandt, O.; Geelhaar, L.; Song, H.; Cho, Y.-H.; Guan, N.; Cavanna, A.; Njeim, J.; Madouri, A.; Barbier, C.; Largeau, L.; Babichev, A.; Julien, F. H.; Travers, L.; Oehler, F.; Gogneau, N.; Harmand, J.-C.; Tchernycheva, M.. Optical Properties of GaN nanowires grown on chemical vapor deposited-graphene. Nanotechnology 2019, 30, 214005.

(41) Li, X.; Cai, W.; An, J.; Kim, S.; Nah, J.; Yang, D.; Piner, R.; Velamakanni, A.; Jung, I.; Tutuc, E.; Banerjee, S. K.; Colombo, L.;

Ruoff, R. S. Large-area synthesis of high-quality and uniform graphene films on copper foils. Science 2009, 324, 1312-1314.

(42) Auzelle, T.; Haas, B.; Den Hertog, M.; Rouvière, J.-L.; Daudin, B.; Gayral, B. Attribution of the $3.45 \mathrm{eV} \mathrm{GaN} \mathrm{nanowires}$ luminescence to inversion domain boundaries. Appl. Phys. Lett. 2015, 107, 051904.

(43) Kumaresan, V.; Largeau, L.; Oehler, F.; Zhang, H.; Mauguin, O.; Glas, F.; Gogneau, N.; Tchernycheva, M.; Harmand, J. C. Selfinduced growth of vertical GaN nanowires on silica. Nanotechnology 2016, 27 (13), 135602.

(44) Acik, M.; Chabal, Y. J. Nature of graphene edges: A Review. Jpn. J. Appl. Phys. 2011, 50, 070101.

(45) Jung, M. W.; Song, W.; Jung, D. S.; Lee, S. S.; Park, C.-Y.; An, K.-S. Tuning the electrical properties of graphene via nitrogen plasma-assisted chemical modification. J. Nanosci. Nanotechnol. 2016, 16, 2756-2759.

(46) Liu, J.; Liang, H.; Xia, X.; Abbas, Q.; Liu, Y.; Luo, Y.; Zhang, Y.; Yan, L.; Han, X.; Du, G. Anomalous Indium Incorporation and Optical properties of high indium content InGaN grown by MOCVD. J. Alloys Compd. 2018, 735, 1239-1244.

(47) Fernández-Garrido, S.; Kaganer, V. M.; Sabelfeld, K. K.; Gotschke, T.; Grandal, J.; Calleja, E.; Geelhaar, L.; Brandt, O. SelfRegulated Radius of spontaneously formed GaN nanowires in molecular beam epitaxy. Nano Lett. 2013, 13, 3274-3280.

(48) Consonni, V.; Dubrovskii, V. G.; Trampert, A.; Geelhaar, L.; Riechert, H. Quantitative description for the growth rate of self-induced GaN nanowires. Phys. Rev. B 2012, 85, 1-7.

(49) Oehler, F.; Cattoni, A.; Scaccabarozzi, A.; Patriarche, G.; Glas, F.; Harmand, J. Measuring and modeling the growth dynamics of selfcatalyzed GaP nanowire arrays. Nano Lett. 2018, 18, 701-708.

(50) Consonni, V.; Knelangen, M.; Geelhaar, L.; Trampert, A.; Riechert, H. Nucleation mechanisms of epitaxial GaN nanowires: origin of their self-induced formation and initial radius. Phys. Rev. B 2010, 81, 1-10.

(51) Sabelfeld, K. K.; Kaganer, V. M.; Limbach, F.; Dogan, P.; Brandt, O.; Sabelfeld, K. K.; Kaganer, V. M.; Limbach, F.; Dogan, P.; Brandt, O.; Geelhaar, L.; Riechert, H. Height self-equilibration during the growth of dense nanowire ensembles: order emerging from disorder height self-equilibration during the growth of dense nanowire ensembles: order emerging from disorder. Appl. Phys. Lett. 2013, $103,1105$.

(52) Fernández-Garrido, S.; Zettler, J. K.; Geelhaar, L.; Brandt, O. Monitoring the formation of nanowires by line-of-sight quadrupole mass spectrometry: a comprehensive description of the temporal evolution of GaN nanowire ensembles. Nano Lett. 2015, 15, $1930-1937$. 
(53) Dubrovskii, V. G.; Sibirev, N. V.; Berdnikov, Y.; Gomes, U. P.; Ercolani, D.; Zannier, V.; Sorba, L. Length distributions of Aucatalyzed and in-catalyzed InAs nanowires. Nanotechnology 2016, 27, 1-9.

(54) Sobanska, M.; Dubrovskii, V. G.; Tchutchulashvili, G.; Klosek, K.; Zytkiewicz, Z. R. Analysis of incubation times for the selfinduced formation of GaN nanowires: influence of the substrate on the nucleation mechanism. Cryst. Growth Des. 2016, 16, 7205-7211.

(55) Vukajlovic-Plestina, J.; Kim, W.; Dubrovskii, V. G.; Tütüncüoğlu, G.; Lagier, M.; Potts, H.; Friedl, M.; Fontcuberta i Morral, A. Engineering the size distributions of ordered GaAs nanowires on silicon, Nano Lett. 2017, 17, 4101-4108.

(56) Mata, R.; Hestroffer, K.; Budagosky, J.; Cros, A.; Bougerol, C.; Renevier, H.; Daudin, B. Nucleation of GaN nanowires grown by plasma-assisted molecular beam epitaxy: the effect of temperature. J. Cryst. Growth 2011, 334, 177-180.

(57) Carnevale, S. D.; Yang, J.; Phillips, P. J.; Mills, M. J.; Myers, R. C. Three-dimensional GaN/AlN nanowire heterostructures by separating nucleation and growth processes. Nano Lett. 2011, 11, 866-871. 


\section{TOC GRAPHIC:}

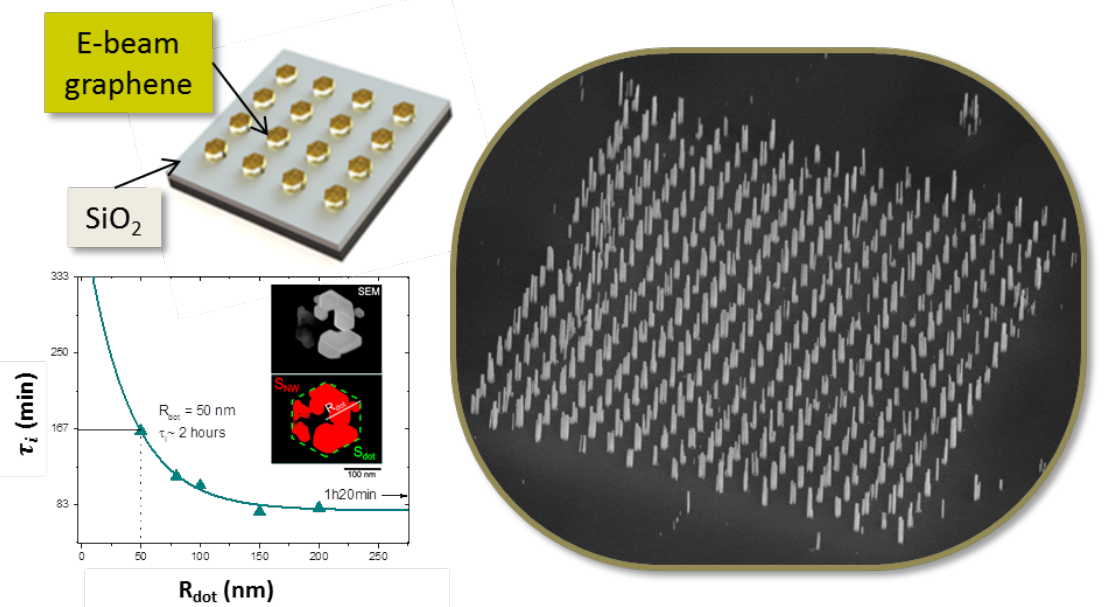

Figure summarizing the main topic and results of the manuscript: growth of nanowires on ebeam lithographed graphene on $\mathrm{SiO}_{2}$ (scheme of the substrate and SEM image) + graphene dot size-dependent NW incubation time. 